\title{
Inhibitory effects of accumbal transmembrane protein 168 (TMEM168) on methamphetamine-induced place
}

\author{
Atsumi Nitta ${ }^{1}$, Kequan $\mathrm{Fu}^{1}$, Kazuya Otake ${ }^{1}$, Kazuyuki Sumi ${ }^{1}$, Eriko Saika ${ }^{1}$, Naoki Sato ${ }^{1}$, Yuka Ueno ${ }^{1}$, \\ Kyosuke Uno ${ }^{1}$, Shin-Ichi Muramatsu ${ }^{2,3}$, Yoshiaki Miyamoto ${ }^{1}$ \\ ${ }^{1}$ University of Toyama, Toyama, Department of Pharmaceutical Therapy and Neuropharmacology, Faculty of \\ Pharmaceutical Sciences, Graduate School of Medicine and Pharmaceutical Sciences, Japan, ${ }^{2}$ Division of Neurology, \\ Department of Medicine, Jichi Medical University, Japan, ${ }^{3}$ Center for Gene and Cell Therapy, Institute of Medical \\ Science, The University of Tokyo, Japan
}

Background: Chronic exposure to methamphetamine causes adaptive changes in brain, which underlie dependence symptoms. We have found that the transmembrane protein 168 (TMEM168) is overexpressed in the nucleus accumbens of mice upon repeated methamphetamine administration.

Methods; We overexpressed TMEM168 in the nucleus accumbens of mice by using an adeno-associated virus vector (NAc-TMEM mice). Locomotor activity and conditioned place preference (CPP) tests were performed to investigate the affection of the pharmacological effects of methamphetamine. Mice were microinjected with OPN (0.03 microgram) or PBS (vehicle) in a total volume of $0.25 \mathrm{microL} /$ side at a rate of $0.25 \mathrm{microL} / \mathrm{min}$ using a microdrive pump. In the In vivo microdialysis test, four hours after the probe was inserted, the baseline of DA levels was measured, which was the mean of the last three samples before METH administration or high pottasium stimulation (100 mM). Golgi in the NAc of mice was isolated by Percoll density gradient centrifugation. Co-immunoprecipitations of TMEM168-flag and OPN were carried out from total cell lysates in 300 microL volume, which was centrifuged. The supernatant was incubated with 3 micro anti-flag antibody.

[Results] Methamphetamine-induced hyperlocomotion and CPP were attenuated in NAc-TMEM mice. Additionally, methamphetamine-induced extracellular dopamine elevation was suppressed in the nucleus accumbens of NAc-TMEM mice. We identified extracellular matrix protein osteopontin as an interacting partner of TMEM168, by conducting immunoprecipitation in cultured COS-7 cells. TMEM168 overexpression in COS-7 cells induced the enhancement of extracellular and intracellular osteopontin. Similarly, osteopontin enhancement was also observed in the nucleus accumbens of NAc-TMEM mice, in in vivo studies. Furthermore, the infusion of osteopontin proteins into the nucleus accumbens of mice was found to inhibit methamphetamine-induced hyperlocomotion and conditioned place preference. Conclusions; Our studies suggest that the TMEM168-regulated osteopontin system is a novel target pathway for the therapy of methamphetamine dependence, via regulating the dopaminergic function in the nucleus accumbens. 